\title{
Modeling the Influence of Temperature-dependent Thermal Properties on the Freezing Front
}

\author{
Victor M. Chavarria ${ }^{1}$ \\ ${ }^{1}$ Food Research Consultant, La Paz, Bolivia \\ Correspondence: Victor M. Chavarria, La Paz, Bolivia. Tel: 591-22752177. E-mail: victor.chavarria@yahoo.com
}

Received: September 25, 2019

Accepted: October 17, 2019 Online Published: October 30, 2019

doi:10.5539/jfr.v8n6p129

URL: https://doi.org/10.5539/jfr.v8n6p129

\begin{abstract}
Although numerical methods enable comprehensive analyses of food freezing, a thorough quantification is lacking the effects on the process introduced by uncertainties in variable thermal properties. Analytical models are, however, more suitable tools to perform such calculations. We aim to quantify these effects by developing a solution to the freezing front (FF) problem subject to temperature-dependent thermal properties and one-dimensional convective cooling. The heat integral balance method, Kirchhoff's transformation, and Plank's cooled-surface temperature equation (as a seed function) enabled us to obtain an approximate solution to the FF penetration time $(\tau)$. To optimize model accuracy, two adjustable parameters were correlated with the inputs via nonlinear regression referenced to numerical simulation FF data. The mapped $(\tau)$ sensitivities, generated by perturbations in the temperature-dependent thermal conductivity and effective heat capacity $\left(\sigma_{\kappa}\right.$ and $\sigma_{\lambda}$, respectively), undergo rapid nonlinear changes for Biot numbers $\leq 6$. Above this level, these sensitivities stabilize depending on the cooling medium temperature and a thermal conductivity parameter. The median $\sigma_{\kappa}$ is 0.348 and its interquartile range (IQR) is 0.220 to 0.425 , whereas the median $\sigma_{\lambda}$ is 0.967 (IQR: 0.877 to 0.985 ). Statistical error measures and a ten-split $K$-fold validation support the model accuracy and reliability of the parameter estimates. Together, the model allows for gaining insights into the nonlinear behavior and magnitude of the influence of variable properties on the FF for a wide range of conditions. Nonlinear methods and prior information enable practical modeling of transport phenomena in foods.
\end{abstract}

Keywords: freezing front, moving boundary, mathematical modeling, perturbation analysis, temperature-dependent thermal properties

\section{Introduction}

The exact mathematical analysis of phase change in foods is largely limited by nonlinearities introduced a set of assumptions comprising temperature-dependent material properties, convective cooling (heating) and finite domains. Because of their multi-component system, structure and stability properties, thermal properties are difficult to measure and model accurately (Heldman \& Singh, 1981). Consequently, it is relevant to understand the impact that uncertainties in physical properties have on product design to energy consumption aspects of industrial freezing operations (Schwartzberg, 1976; Pham \& Willix, 1990).

Invoking the moving boundary (MB) concept reduces the complexity of the phase change problem by formulating one or more material regions separated by well-defined interfaces and subject to simplified physical assumptions. For example, pseudo-steady state and constant thermal property assumptions were central to Plank's analysis of freezing times of food materials (López-Leiva \& Hallström, 2003). Analytic solutions of the freezing front (FF) with variable thermal properties are available only for limited cases (Kucera, 1985; Lunardini, 1988; Ramos, Cerrato \& Gutierrez, 1994; Voller, Svenson \& Paola, 2004; Singh, Gupta \& Ral, 2011; Rajeev, 2014; Kumar \& Singh, 2018). Specifically, the problem becomes analytically intractable when formulated with convective cooling, finite geometry, gradual heat release (absorption), and temperature-dependent thermal property models with meaningful physical parameters (Özisik, 1980; Delgado \& Sun, 2001; Schwartzberg, Singh \& Sarkar, 2007).

Although numerical models are suitable to solve complex phase change problems, analytic models are more efficient and compact to understand the specific roles that variable thermal properties play in the process (Özisik, 1980). The central objective of this exploratory research is to quantify and characterize the effects of variable thermal properties on the freezing front. To accomplish this aim, we apply nonlinear methods to analyze the FF problem subject to the 
aforementioned formulation. Our modeling strategy includes (1) solution to the FF problem using nonlinear methods and prior information characterizing the convective surface temperature, (2) FF data generation via numerical simulation, (3) nonlinear-regression estimation of two adjustable parameters to optimize model accuracy, (4) implementation of the $K$-fold model validation, and (5) quantification and mapping of the effects of temperature-dependent thermal properties on the FF.

\section{Literature Review}

The MB formulation has been applied to processes like food freezing, thawing, frying, ablation, bubble growth, sedimentation, and shoreline movement (Kucera, 1985; Farid, 2002; Voller et al., 2004; Rajeev, 2014). This partial review identifies analytic methods used in heat conduction problems with moving boundaries characterized by the Stefan condition.

Throughout this paper, we refer to the following assumptions:

- A1, constant thermal properties for each phase;

- A2, Dirichlet, fixed cooling medium temperature, boundary condition (BC);

- A3, Robin BC with constant medium temperature;

- A4, Neumann BC with constant surface heat flux;

- A5, semi-infinite domain;

- A6, one-dimensional finite geometry;

- A7, initial temperature at the initial freezing point;

- A8, no sensible heat release;

- A9, total latent heat release at a unique temperature;

- A10, pseudo-steady state (PSS), for Stefan values smaller than 1; and

- A11, temperature-dependent thermal properties and gradual heat release (absorption).

Although we review some relevant solutions to illustrate their functional expressions, we focus on the potential analytical methods to solve the MB problem with conditions A3, A6, A7, and A11. An in-depth theoretical treatment of the Stefan problem can be found elsewhere (Goodman, 1958; Solomon, Wilson \& Alexiades, 1981; Kucera, 1985; Lunardini, 1988; Mitchell \& Myers, 2012).

Neumann applied similarity transformation to a two-phase freezing problem with assumptions A1, A2, A5, and A9 (Solomon et al., 1981). His exact solution describes the moving boundary $(X(t))$ being proportional to $\sqrt{t}$. This square-root of time relation is a well-known result of MB physics. By further imposing assumption A10, the similarity solution becomes $X(t)=\sqrt{2 \mathrm{St} \alpha_{f} t}$. If thermal properties and gradual latent heat release are temperature dependent, the FF motion may depart from the $\sqrt{t}$ law (Bazant, 2018). The set of assumptions behind Plank's freezing time Eq. (1) includes A1, A3, A6 to A10 (Hu \& Argyropoulos, 1996). In addition to small Stefan numbers, assumption A10 also requires negligible diffusion in the freezing region (Kucera, 1985; Lunardini, 1988). Several modifications of Eq. (1) have been developed to compensate for Plank's simplified set of assumptions (Pham, 1986; Mittal, Hanenian \& Mellikarjunan, 1993; Delgado \& Sun, 2001; Fricke \& Becker, 2001). Plank's freezing time equation is given by:

$$
\tau_{P k}=\frac{s(\tau)}{\mathrm{Bi} \cdot \mathrm{St}}(0.5 \mathrm{Bi} s(\tau)+1),
$$

where $s(\tau)$ is the dimensionless FF, $\tau$ the dimensionless time, Bi the Biot number, and St the Stefan number; all based on the frozen-state material properties.

Considering that St is less than 1 for most foodstuffs (Schwartzberg, 1976), its effect is to slow down the diffusion process. Kucera (1985) applied boundary fixing transformation and iterative series methods to the Stefan (solidification) problem invoking assumptions A1, A4, A5, A7, A9, and A10. The upper-bound FF penetration time expression given by Kucera is:

$$
\tau_{\mathrm{Kc}}=\tau_{\mathrm{Pk}}+s(\tau)^{2} \frac{(3+\mathrm{Bi} s(\tau))}{6(1+\mathrm{Bi} s(\tau))},
$$

where $\tau_{\mathrm{Pk}}$ is estimated from Eq. (1), and valid for long times $\tau \geq \mathcal{O}\left[\mathrm{St}^{-1}\right]$. 
Goodman's (1958) melting analysis implemented the heat balance integral (HBI) method with assumptions A1, A4, A5, A7, and A9. Several HBI investigations have addressed the Stefan problem with assumption A1, A3 or A4, A6, and A9 using polynomial, exponential or trigonometric temperature profiles (Özisik, 1980; Wood, 2001; Mitchell \& Myers, 2012). The latter authors recommended a cubic polynomial for HBI analysis of melting problems as optimum modeling strategy. This HBI volume-averaging method allows removing the temperature variable from the energy partial differential equation (PDE) and reducing it to an ordinary differential equation (ODE). Özisik (1980, p. 419) applied similarity variable transformation to the frozen phase and HBI to the freezing phase using assumptions A1, A2, A6, and A7. His solution requires, however, solving a transcendental equation and specifying a temperature-polynomial degree. Baudouy (2002) implemented Kirchhoff's transformation and HBI methods for heat transfer in Helium II with assumptions A2 and A5. It has been proven that for $c_{\mathrm{p}} \approx 0$, the solution to the Stefan problem with assumptions A1, A3, and A6 to A9 approaches the PSS solution (Solomon et al., 1981). Together, the aforementioned methods, however effective in obtaining approximate FF solutions, did not solve the phase change problem considering assumptions A3, A6, A7, and A11.

Variational iteration (Singh et al., 2011) and homotopy perturbation (Rajeev, 2014) methods were applied to space-variable physical properties and latent heat in Stefan problems. Ramos et al. (1994) implemented the group transformation theory to solve for transient temperatures during freezing (thawing). Their assumptions A3, A6, and A7 also included a variable effective heat capacity (albeit difficult to interpret) and thermal conductivity described, however, by empirical power-law models.

Cho and Sunderland (1974) used a modified error function to solve the Neumann problem considering a linearly temperature-dependent thermal diffusivity. The effect on the moving boundary was significant only for thermal diffusivity with marked temperature dependence and for large Stefan numbers (St 》 1). Kumar and Singh (2018) found comparable results by applying similarity variable transformation and Chebyshev polynomials. Their assumptions comprised A2, A5, specific heat and thermal conductivity properties that followed assumed empirical power-law functions of temperature.

Heldman and Singh (1981) described the influence variable thermal properties on temperature fields applying numerical and experimental methods but without systematic analysis of errors in thermal property data. This type of analysis has been addressed relying on (semi) empirical freezing-time correlations and yielding only mean effects for a narrow range of input physical parameters (Mittal et al. 1993; Fricke \& Becker, 2001; Rao, Rizvi \& Datta, 2005). Whereas the reported sensitivities due to perturbations in frozen thermal conductivity $\left(\sigma_{\kappa}\right)$ vary from \pm 0.01 to \pm 0.15 , the sensitivity driven by latent heat perturbations $\left(\sigma_{\lambda}\right)$ is approximately 1.16 . Pham and Willix (1990) conducted an error analysis of process times due to Biot number deviations, but not thermal properties. Mittal et al. (1993) evaluated the influence of errors associated with thermal properties on the freezing process considering twelve well-known freezing-time (semi) empirical correlations. The latter are essentially modifications of Plank's Eq. (1).

Collectively, a specific understanding of the role of variable temperature-dependent thermal properties on the freezing front is not thorough and has thus motivated this investigation. An analytical and accurate FF model based on realistic assumptions would enable such quantification. The advantages of such a model over numerical solutions include no computer programming, no numerical convergence or stability problems, and straightforward calculations of the effects of variable thermal properties on the MB. Additionally, the model could be used as a convenient educational tool to describe the FF in food materials.

\section{Methods}

Our analytical strategy comprised HBI and Kirchhoff's transformation methods and a simple convective surface-temperature expression as a seed function. Ideally, the analysis would yield an FF solution that leads to an improved temperature equation. The iterative process is repeated until it converges. If the functional complexity of the updated surface-temperature expression curtails the iteration process, the accuracy of the first solution is optimized by nonlinear regression of adjustable parameters introduced in the seed function.

\subsection{Numerical Models}

Strategy steps (1) to (3) relied on Wolfram Mathematica's 11 symbolic, NDSolve (Method of Lines), and NonlinearModelFit functions for analysis, numerical simulation, and nonlinear regression calculations, respectively. Mathematica's least-squares minimization uses the Levenberg-Marquardt algorithm. A numerical model implemented the finite difference Crank-Nicolson time-stepping scheme (CNFD) and the Thomas algorithm in MS Excel 10 to solve the phase change problem (Carnahan, Luther \& Wilkes, 1969). This model allowed for generating one-dimensional transient temperatures and FF position-time data. We validated the 
CNFD model against the exact series solution of the heat conduction assuming constant thermal properties (Özisik, 1980). CNFD-model freezing predictions were also compared to Mathematica's numerical temperature solution and to Pham and Willix's (1990) experimental freezing times for tylose substance. The latter validation yielded a Pearson correlation coefficient of 0.998 ( $p *<0.01)$.

\subsection{Statistical Error Analysis and Validation}

Error analysis of nonlinear regression in step (3), fit quality evaluation and selection of correlation models followed published methodologies (Beck \& Arnold, 1977; Archontoulis \& Miguez, 2015). Mathematica's Generalized Linear Model (GLM) enabled quantifying the significance of thermal property effects on FF penetration-time sensitivities. To validate the final parameterized model in step (4), we implemented a ten split $K$-fold method with a 90:10 data partition. The model training and error validation sets comprised 1350 and 150 data points, respectively. The final model accuracy is an average of ten validation $K$-fold error estimates (Arboretti-Giancristofaro \& Salmaso, 2003; Jung \& Hu, 2015).

\subsection{Thermal Property Models}

To quantify the influence of variable thermal properties on the FF in step (5), we adopted Schwartzberg's (1976) thermal conductivity $(\hat{k})$ and effective heat capacity $\left(\hat{c}_{p}\right)$ models. Below the initial freezing point, the normalized properties are given by $\hat{k}(\theta)=1-\kappa /(1-\theta)$ and $\hat{c}_{p}(\theta)=1+\lambda /(1-\theta)^{2}$. Each model depends on a single thermal-property parameter that controls their temperature dependence. Estimation of $\kappa$ and $\lambda$ is straightforward using published thermal property data. These models provide reliable estimates of $\hat{k}$ and $\hat{c}_{p}$ applicable to a practical range of food types and industrial freezing conditions (Schwartzberg, 1977). Further, the models have monotonic and differentiable properties over the entire freezing temperature range (Figure 1).

\subsection{Freezing Front Calculation}

Rather than an infinitesimal thin interface, the FF that develops in multi-component foods occurs as a progressive, yet brief, decrease in temperature away from the initial freezing point (Farid, 2002). See the curved end of the plateau (top shadow) of the temperature surface in Figure 2. The FF location was computed as a time-heat event from simulated transient-temperature fields. When the normalized and accumulated heat release (relative to the initial material heat content) changes by a minute but detectable amount or threshold, it signals the front's transition. This detection limit was set equal to $0.334 \%$, considering the total temperature measurement error, which comprises thermocouple probe, instrumentation, and calibration errors (Cottrell, 2006).

\subsection{Freezing Front Penetration-time Sensitivity}

To map the effects of variable $\hat{k}$ and $\hat{c}_{p}$, we generated perturbations in the single property parameter ( $\kappa$ or $\lambda$ ) that controls their temperature dependence. By introducing a relative error (uncertainty) in $\kappa\left(\hat{\epsilon}_{\kappa}= \pm 3 \%\right)$, keeping $\lambda$ constant, the effect on FF penetration time was calculated as a sensitivity measure $\left(\sigma_{\kappa}\right)$ equal to $\hat{\epsilon}_{\tau} / \hat{\epsilon}_{\kappa}$. The same procedure applied to perturbations in $\lambda$ to calculate $\sigma_{\lambda}$ as $\hat{\epsilon}_{\tau} / \hat{\epsilon}_{\lambda}$. The simultaneous effect of $\kappa$ and $\lambda$ perturbations on FF $\left(\sigma_{\kappa \lambda}\right)$ was computed using Monte Carlo simulation with 5000 iterations and assuming an $\mathrm{SD}$ of $\pm 3 \%$ of the property parameter means. Under the law of propagation of uncorrelated and random errors, the joint sensitivity becomes (Tellingheusen, 2001):

$$
\sigma_{\kappa \lambda}=\sqrt{\frac{\left(\sigma_{K} \widehat{\varepsilon_{\kappa}}\right)^{2}+\left(\sigma_{\lambda} \widehat{\varepsilon_{\lambda}}\right)^{2}}{\widehat{\varepsilon}_{\kappa}^{2}+\hat{\varepsilon}_{\lambda}^{2}}}
$$

where $\sigma_{\kappa}$ is $\widehat{\varepsilon}_{\tau} / \sqrt{\widehat{\varepsilon}_{\kappa}{ }^{2}+\widehat{\varepsilon}_{\lambda}^{2}}$.

\subsection{Numerical Test Design}

Test grids in Table 1 were designed to control the following input factors: $\mathrm{Bi}, \lambda, \psi, \kappa$, and $s$ in the numerical computations (Chavarria, 2019). Whereas grid T1.1 was used for parameters $(n)$ and $(m)$ calculations, grid T1.2 applied to computation of $\sigma_{\lambda}$ and $\sigma_{\kappa}$ penetration time sensitivities. Test grid T1.2 was defined as full factorial. In turn, test grid T1.1 was designed considering $1 / \mathrm{Bi}^{2}$ and St effects on FF. The sample size $(N)$ for grid T1.1 was estimated following Bellera and Hanley (2007). The sample points were defined according to the following cumulative (percentage) distribution with respect to $\mathrm{Bi} \cdot$ St levels:

$\{\{<0.015,55.1 \%\},\{0.45,69.6 \%\},\{0.8,73.5 \%\},\{1.5,80.2 \%\},\{2.55 .88 .5 \%\},\{3.95,92.5 \%\},\{6.05,94.9 \%\}$,

$\{8.15,96.7 \%\},\{10.25,99.0 \%\},\{11,3,100 \%\}\}$. 
Table 1. Numerical experimentation test design

\begin{tabular}{|c|c|c|c|c|c|c|c|c|c|}
\hline \multirow{2}{*}{$\begin{array}{l}\text { Analysis } \\
\text { Grid T1.1 }\end{array}$} & \multirow{2}{*}{$\begin{array}{l}\text { Factor } \\
\lambda\end{array}$} & \multicolumn{8}{|c|}{ Levels } \\
\hline & & & & $95^{*}$ & $105^{*}$ & $115^{*}$ & & & \\
\hline$n$ and $m$ & $\psi$ & & -40 & -32 & -24 & -16 & -8 & & \\
\hline parameter & $\kappa$ & & & $0.4^{*}$ & $0.5^{*}$ & $0.6^{*}$ & & & \\
\hline estimation, & $s$ & 0 & 0.4 & 0.5 & 0.5 & 0.8 & 0.9 & 1 & \\
\hline$N=1500$ & $\mathrm{Bi}$ & 0.06 & 0.061 & 0.062 & 0.0634 & 0.64 & 0.65 & 0.0667 & 0.07 \\
\hline & & 0.0734 & 0.0767 & 0.080 & 0.834 & 0.090 & 0.093 & 0.967 & 0.30 \\
\hline & & 0.40 & 0.60 & 1 & 3 & 6 & 10 & 25 & \\
\hline Grid T1.2 & $\lambda$ & & & 90 & 100 & 110 & 120 & & \\
\hline$\sigma_{\mathrm{K}}, \sigma_{\lambda}$ & $\psi$ & & -40 & -32 & -24 & -16 & -8 & & \\
\hline$\sigma_{\mathrm{K} \lambda}$ & $\kappa$ & & & 0.4 & 0.5 & 0.6 & & & \\
\hline calculations & $\mathrm{Bi}$ & 0.06 & 0.07 & 0.08 & 0.09 & 0.1 & 0.14 & 0.18 & \\
\hline$N=1200$ & & 0.22 & 0.27 & 0.35 & 0.45 & 0.60 & 1.5 & 3 & \\
\hline & & 6 & 10 & 13 & 17 & 21 & 25 & & \\
\hline Grid T1.3 & $\lambda$ & & & & 105 & & & & \\
\hline$\sigma_{\mathrm{K} \lambda}$ & $\psi$ & & -40 & -32 & -24 & -16 & -8 & & \\
\hline mapping & $\kappa$ & & & & 0.5 & & & & \\
\hline$N=45$ & $\mathrm{Bi}$ & 0.06 & 0.09 & 0.667 & 1.25 & 3 & 6 & 12 & \\
\hline & & 18 & 25 & & & & & & \\
\hline
\end{tabular}

Test grids for the computation of the final model parameters and sensitivities generated by thermal property perturbations. Levels with an asterisk indicate additional values in the factor neighborhood

\section{Analysis}

\subsection{Freezing Front Derivation}

The one-dimensional MB assumes a virtual interface or surface separating the frozen phase from the freezing region across the slab thickness and moving at an unknown velocity towards the thermal center (Farid, 2002). This interface is defined by the Stefan condition that (a) links both temperature fields, (b) requires the rate of latent heat removal to balance the heat flux into the freezing region, and (c) forces the temperature continuity across the boundary (Kucera, 1988). The analytical task is to solve for both the transient thermal fields in each region and the FF location.

In the integral method, all the heat phenomena take place within a thermal layer that grows from the cooled surface towards the slab center. This dynamic depth ends where the heat flux is zero and the temperature remains at the initial phase change point. Once the slab center is reached, the thermal layer concept vanishes (Özisik, 1980).

The one-dimensional heat conduction PDE, which accounts for temperature-dependent thermal conductivity and effective heat capacity, but no density change, is given by (Heldman \& Singh, 1981; Schwartzberg et al., 2007):

$$
\hat{c}_{p}(\theta(r, \tau)) \frac{\partial \theta(r, \tau)}{\partial \tau}=\frac{\partial}{\partial r}\left[\hat{k}(\theta(r, \tau)) \frac{\partial \theta(r, \tau)}{\partial r}\right],
$$

where the dimensionless spatial coordinate is $r=x / L$. The initial condition is $\theta(r, 0)=0$, whereas at very long times $\theta(r, \tau \rightarrow \infty)=\psi$.

The BCs are:

$$
\begin{gathered}
\partial_{r} \theta(r, \tau)=0 \quad \text { at } r=1, \\
-\hat{k}\left(\theta_{w}(r, \tau)\right) \partial_{r} \theta(r, \tau)=\mathrm{Bi}\left(\psi-\theta_{w}(\tau)\right) \quad \text { at } r=0,
\end{gathered}
$$

where $\theta_{\mathrm{w}}(\tau)=\theta(0, \tau)$.

Applying Kirchhoff's transformation to the $\hat{c}_{\mathrm{p}}(\theta)$ model, yields the new temperature $u$ (Özisik, 1980):

$$
u=\int_{0}^{\theta} \hat{c}_{\mathrm{p}}(\theta) d \theta=\theta+\theta \lambda /(1-\theta) .
$$


If Eq. (7) is plugged into (4), we obtain:

$$
\frac{\partial u(r, \tau)}{\partial \tau}=\frac{\partial}{\partial r}\left[\widehat{\alpha}(\theta(r, \tau)) \frac{\partial u(r, \tau)}{\partial r}\right]
$$

where $\widehat{\alpha}(\theta)=\hat{k}(\theta) / \hat{c}_{p}(\theta)$ becomes an apparent diffusivity term that captures the nonlinear temperature effects. The $r$ domain in the thermal layer ranges now from 0 to $s(\tau)$. Whereas the initial condition is $u(r, 0)=0$, the BCs are:

$$
\begin{array}{cc}
u(r, \tau)=0 \text { and } \partial_{r} u(r, \tau)=0 & \text { at } r=s(\tau), \\
-\hat{\alpha}\left(\theta_{w}(\tau)\right) \partial_{r} u(r, \tau)=\operatorname{Bi}\left(\psi-\theta_{w}(\tau)\right) & \text { at } r=0 .
\end{array}
$$

The RHS of Eq, (10) is a prescribed function of the convective heat flux in terms of $s$ (short notation for $s(\tau)$ ) and is only a function of time. Therefore, it requires no Kirchhoff's transformation (Özisik, 1980). If Eq. (8) is integrated over $r$, from 0 to $s$, the HBI equation becomes:

$$
\int_{0}^{s} \partial_{\tau} u(r, \tau) d r=\widehat{\alpha}(\theta) \partial_{r} u(s, \tau)-\widehat{\alpha}(\theta) \partial_{r} u(0, \tau),
$$

where the first term on the RHS vanishes with the zero heat flux at $r=s(\tau)$. Applying Leibniz' integration rule together with $u(s, \tau)=0$ to the LHS and Eq. (10) to the RHS of Eq. (11) yields:

$$
\partial_{\tau} \int_{0}^{s} u(r, \tau) d r=\operatorname{Bi}\left(\psi-\theta_{\mathrm{w}}(\tau)\right)
$$

To eliminate $\theta_{w}$ in Eq. (12), we invoke the following convective-surface temperature function:

$$
\theta_{\mathrm{w}}(s)=\operatorname{Bi} m s \psi /(1+\operatorname{Bi} m s),
$$

which can be readily derived from Eq. (1) setting $m=1$.

Even though Eq. (13) is a simple rational expression, its introduction as a seed function generates a solution with greater functional properties. Goodman (1958, p. 339) obtained a similar equation for $\mathrm{St}=0$; but even for $0<\mathrm{St}$ $<1$, it can be shown that his equation still holds with a relative error of $1.2 \%$. For food freezing processes, St values vary from 0.04 to 0.45 (Schwartzberg, 1977). Parameter $m$ was introduced into Eq. (13) to account for Bi and St effects considering Goodman's analysis.

A temperature polynomial of $n$-th degree that satisfies Eq. (9) is given by:

$$
u(r, \tau)=u_{w}(\tau)(1-r)^{n}
$$

Evaluating the $u$ integral on the LHS of Eq. (12) with Eq. (14) yields:

$$
\int_{0}^{s} u(r, \tau) d r=\frac{s u_{w}(s)}{1+n} .
$$

If Equations (13) and (15) are plugged into (12), the HBI equation becomes:

$$
\frac{\partial}{\partial \tau}\left[\frac{s u_{W}(s)}{1+n}\right]=\frac{\operatorname{Bi} \psi}{1+\operatorname{Bi} m s} .
$$

Combining Equations (13) and (14) with (10), recasts the energy balance at the cooled surface as:

$$
\frac{-n \widehat{\alpha}\left(\theta_{w}(\tau)\right) u_{w}(s)}{s}=\frac{\operatorname{Bi} \psi}{1+\operatorname{Bi} m s} .
$$

Using the expressions of $\widehat{k}(\theta)$ and $\hat{c}_{p}(\theta)$ in $\widehat{\alpha}(\theta)$, we solve for $u_{w}$ from Eq. (17):

$$
\left.u_{w}(s)=\frac{\operatorname{Bi} s \psi}{g_{1}(s)}\left[(\operatorname{Bi} m s)^{2}(\lambda+(\psi-1))^{2}\right)+2 \operatorname{Bi} m s(\lambda-\psi+1)+\lambda+1\right],
$$

where $g_{1}(s)$ is:

$$
g_{1}(s)=n(\operatorname{Bi} m s+1)(1-\operatorname{Bi} m s(\psi-1))(\operatorname{Bi} m s(\kappa+\psi-1)+(\kappa-1)) .
$$

Plugging Eq. (18) into (16) and applying the chain rule of differentiation results in: 


$$
\int_{0}^{\tau} d \tau=\int_{0}^{s} \frac{s}{g_{2}(s)}\left[-2(\kappa-1)(\lambda+1)+(\operatorname{Bi} m s)^{5}(\psi-1) \psi_{1}\left(\lambda+(\psi-1)^{2}\right)\right.
$$

$+2(\operatorname{Bi~} m s)^{4}\left[\kappa(2 \psi-3)+3-4 \psi+\psi^{2}\right)\left(\lambda+(\psi-1)^{2}\right]+\operatorname{Bi} m s[\kappa(\lambda(\psi-9)+7 \psi-9)+\lambda(9-2 \psi)-8 \psi+9]$

$\left.\left.+(\operatorname{Bi} m s)^{3}\left[-2(\psi-1)^{2}(4 \psi-7)+\lambda\left(14-12 \psi+\psi^{2}\right)+\kappa\left(2 \lambda(3 \psi-7)+3 \psi^{3}\right)-19 \psi^{2}\right)+30 \psi-14\right)\right]$

$\left.+4(\operatorname{Bi~} m s)^{2}\left(-2 \lambda(\psi-2)+3 \psi^{2}-7 \psi+4\right)+\kappa\left(\lambda(\psi-4)-2\left(\psi^{2}-3 \psi+2\right)\right)\right] d s$,

where $g_{2}(s)$ is given by:

$$
g_{2}(s)=(n+1)(1+\operatorname{Bi} m s)(1-\operatorname{Bi} m s(\psi-1))^{2}\left(\kappa-1+\operatorname{Bi} m s \psi_{1}\right)^{2},
$$

and $\psi_{1}=\psi-1+\kappa$. Integrating Eq. (19) yields the FF penetration time $(\tau)$ as a function of $(s)$ in Eq. (20):

$$
\begin{gathered}
\tau=\frac{1}{n(1+n) \mathrm{Bi}^{2} \kappa m^{2}}\left(\frac{(-1+\kappa)\left(\kappa^{2}+\lambda\right)}{\psi_{1}^{3}}\left(\frac{\operatorname{Bi} m s \psi \psi_{1}}{\psi_{1} \operatorname{Bi} m s+\kappa-1}+\operatorname{Ln}\left[1+\frac{\psi_{1} \operatorname{Bi} m s}{(-1+\kappa)}\right]\right)+\frac{\kappa \operatorname{Bi} m s\left(\lambda+(-1+\psi)^{2}\right)}{2(-1+\psi) \psi_{1}}(2+\operatorname{Bi} m s)\right. \\
\left.-\kappa \operatorname{Ln}[1+\operatorname{Bi} m s]+\frac{\lambda}{(-1+\psi)^{3}}\left(\frac{\operatorname{Bi} m s \psi(-1+\psi)}{(-1+\operatorname{Bi} m s(-1+\psi))}-\operatorname{Ln}[1-\operatorname{Bi} m s(-1+\psi)]\right)\right) .
\end{gathered}
$$

Equation (20) does not explicitly cast the FF $(s)$ in terms of $\tau$, which precludes an updated surface-temperature to be obtained together with further iterations. Goodman's solution (1980, p. 338) is also an implicit expression in $s$. Such functional relation mirrors temperature measurements in food freezing experiments. Sensing probes cannot directly detect the FF. Instead, the time is measured when the temperature barely deviates from the initial freezing point at specific and known slab depths.

The domain of the FF Eq. (20) is $0 \leq s \leq 1$, gives $s(0)=0$, and has a monotonically decreasing derivative $(d s / d \tau>$ 0 ) as is characteristic of one-dimensional MB solutions (Özisik, 1980; Kucera, 1985; Ramos et al. 1994). Whereas $1 / \mathrm{Bi}^{2}, \psi$, and $1 / \mathrm{m}^{2}$ significantly influence $\tau$ by changing its order of magnitude, $\lambda$ and $\kappa$ have only a weak to moderate scaling effect. The limit of $s$ as Bi approaches infinity is $\sqrt{2 n(n+1) \tau}$. This is consistent with the classic HBI solution considering assumptions A1, A2, A5 and A9 (Mitchell \& Myers, 2012). As the parameter $(m)$ approaches zero, the FF time profile shifts from a power law to linear time dependence. The temperature-polynomial power $(n)$ has a linear scaling effect on $\tau$, but it does not impact the freezing-front time profile. All rational, linear, and logarithmic terms in Eq. (20) are significant. Noteworthy is the change of the $s$ vs. $\tau$ curvature at about $0.3<s<0.5$, where it slightly straightens for $(-10<\psi<-8)$ and $(0.06<\mathrm{Bi}<0.1)$. This subtle FF behavior affects the value of $\tau$ at $s=1$ to some degree, but more so the sensitivity $\left(\sigma_{\lambda}\right.$ or $\left.\sigma_{\kappa}\right)$ values, and is addressed in the Discussion.

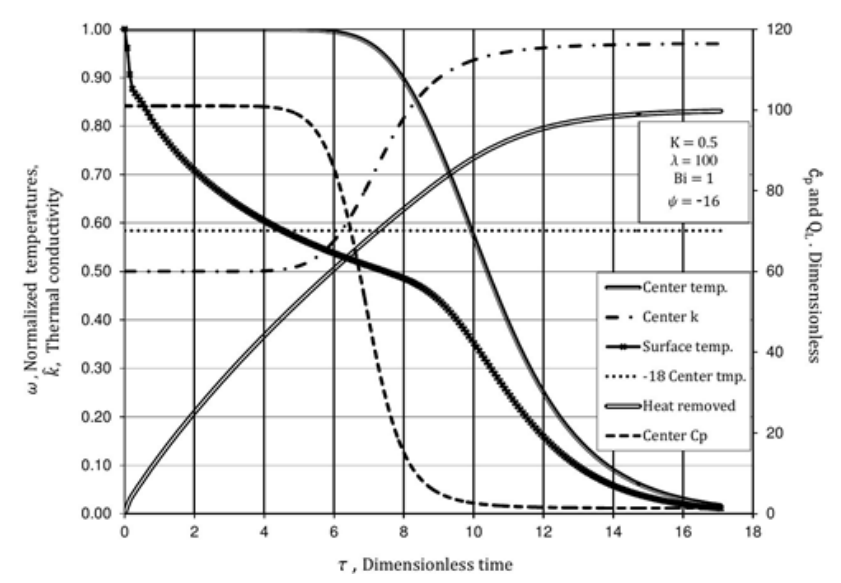

Figure 1. Example of a finite-difference model simulation of normalized slab temperatures, thermal properties, and accumulated heat release below the initial freezing point

\section{Results}

A slab freezing-simulation case, solved for the normalized temperature $(\hat{v})$ using Mathematica's NDSolve function and the CNFD-Excel model, is presented in Figures 1 and 2, respectively. The latter graph also shows the normalized thermal properties $\left(\hat{k}\right.$ and $\left.\hat{c}_{p}\right)$ and the accumulated heat released $\left(\mathrm{Q}_{\mathrm{L}}\right.$.) Note that, for 
this specific case, $\mathrm{Q}_{\mathrm{L}}$ is approximately $62 \%$ when the $\mathrm{FF}$ reaches the slab center at $\tau \approx 6$. The accumulated heat release is, though, always incomplete for the conditions in grid T1.1. As a consequence, with everything else being equal except the gradual heat release, the FF will reach the center faster than the Plank-predicted FF penetration time (Figure 5).

\subsection{Model Optimization by Parameter Estimation: Fit Quality}

Tables 2 and 3 summarize regression results for fitting Eq. (20) with constant $(\hat{n})$ and $(\hat{m})$ values. Notice the relative standard errors are less than $2.8 \%$. The maximum intrinsic $(0.01720)$ and maximum parameter-effects $(0.03384)$ curvatures are less than $6 \%$ of the $95 \%$ confidence region curvature $(0.57718)$. The fit response (lower plot in Figure 3) shows a regression quality higher for slow cooling rates $(\tau>7)$ than for fast rates $(\tau<7)$. This fit inconsistency is reflected in the high relative mean and median errors (Table 3$)$.

To improve model accuracy, parameters $(n)$ and $(m)$ are developed as correlations in terms of input $\mathrm{Bi}$, St, and $\kappa$. Because the term $\left(1 /\left(n(1+n) \mathrm{m}^{2}\right)\right.$ strongly affects Eq. (20) as one scaling factor, the following models are proposed to minimize dependence between the sensitivity coefficients of sub-parameters $n_{1}, n_{2}$ and $m_{1}$ :

$$
\begin{gathered}
n=n_{1}\left(1+\kappa \mathrm{Bi}^{n_{2}}\right) \\
m=n_{2}^{2} /\left(\mathrm{Bi}^{n_{2}}(1+(1+S t) \kappa \mathrm{Bi})^{m_{1}}\right)
\end{gathered}
$$

\subsection{Objective Function Minimization and Parameter Uniqueness}

To verify the least-squares global minimum, we evaluated the SSE using eight levels of each sub-parameter ( $w_{1}$ $\times$ sub-parameter), where $w_{1}$ is: $\{0.1,0.2,0.4,1.11,1,2.5,5,10\}$. Results support the SSE values corresponding to the global minimum least-squares criterion (Table 3). Insofar as parameter uniqueness, regressions were tested with six different initial guesses of each sub-parameter $\left(w_{2} \times\right.$ sub-parameter), where $w_{2}$ is: $\{0.01,0.08,0.64,1$, $4,10\}$. Consistent convergence to the reported estimates and errors in Tables 2 and 3 were obtained as long as the initial values met the following conditions: $1<n_{1}<10, n_{2} \leq 4$, and $m_{1}<0.6$. Outside these limits, the initial guesses led to SSE values greater than the minimized regression error value; to suboptimal SSE values or meaningless negative and nil $n$ values.

\subsection{Parameter Reliability, Residuals and Model Validation}

The pairwise coefficients in the upper-right triangle of the correlation matrix are $\{1,-0.497,-0.177 ; 1,0.665 ; 1\}$. The sensitivity coefficient pairing $n_{2}$ and $m_{1}(0.664)$ indicates partial inter-dependence but only on the second half of the FF depth. Further, the maximum intrinsic (0.00615) and maximum parameter-effects $(0.01019)$ curvatures are approximately two orders of magnitude smaller than the $95 \%$ confidence region curvature (0.61889). These measures confirm the asymptotic and almost-linear behavior of the estimates computed at the converged sub-parameter values and, thus, their reliability (Karolczak \& Mickiewicz, 1995; Seber \& Wild, 2003). The residuals (upper-left corner in Figure 3) are non-normally distributed according to the Jarque-Bera, skewness, and kurtosis tests, whereas the W/S, D'Agostino and Anderson-Darling tests yield opposite results (Ghasemi \& Zahediasl, 2012). This aspect is addressed in the Discussion.

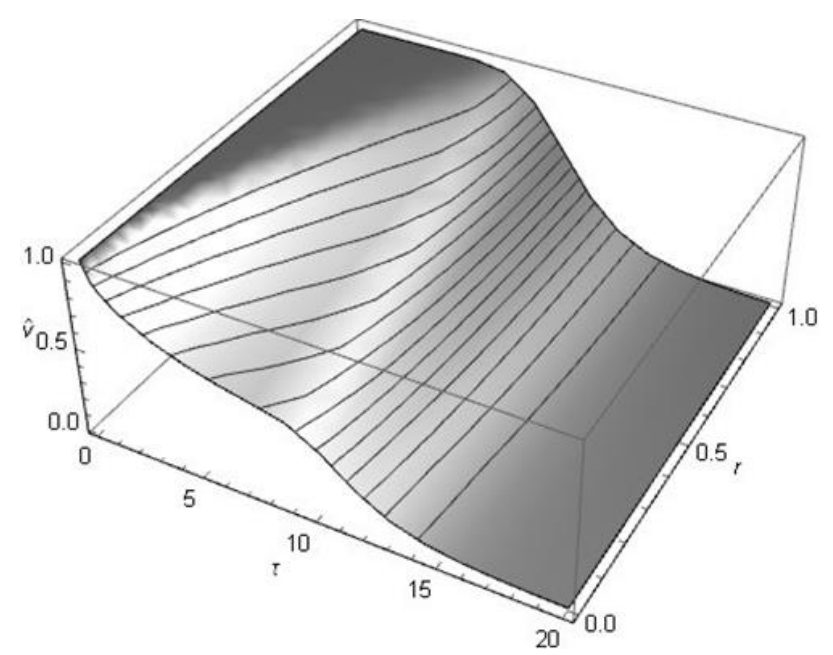

Figure 2. Example of normalized-transient temperature profiles during freezing as predicted by Mathematica's NDSolve kernel function, with $\mathrm{Bi}=1, \psi=-16, \kappa=0.5$ and $\lambda=100$ 
Table 2. Nonlinear regression results estimates and fit measures for $n, m$, and joint sensitivity $\left(\sigma_{\kappa} \lambda\right)$

\begin{tabular}{|c|c|c|c|c|c|c|}
\hline Parameter & Estimate & Standard error & \multicolumn{2}{|c|}{$95 \%$ Conf. interval } & $\mathrm{t}-\mathrm{value}$ & B i a s $(\%)$ \\
\hline$\widehat{n}$ & 3.0826 & 0.00863 & 3.0656 & 3.0995 & 357.2 & $-1.08 \times 10^{-3}$ \\
\hline$\widehat{m}$ & 0.02992 & 0.00068 & 0.0286 & 0.03124 & 44.2 & $7.36 \times 10^{-4}$ \\
\hline \multicolumn{7}{|c|}{ Constant parameters model } \\
\hline$n_{1}$ & 2.2264 & 0.00160 & 2.2233 & 2.2296 & 1381.7 & $-2.48 \times 10^{-4}$ \\
\hline$n_{2}$ & 0.18282 & 0.00064 & 0.1816 & 0.1841 & 285.4 & $-8.84 \times 10^{-6}$ \\
\hline$m_{1}$ & 0.84361 & 0.00579 & 0.8322 & 0.8549 & 145.6 & $1.65 \times 10^{-3}$ \\
\hline \multicolumn{7}{|c|}{ Adjustable parameters model } \\
\hline$c_{1}$ & 0.6558 & 0.00065 & 0.6543 & 0.6589 & 1012.3 & $-9.82 \times 10^{-8}$ \\
\hline$c_{2}$ & 0.0809 & 0.00049 & 0.0799 & 0.0819 & 163.9 & $4.31 \times 10^{-8}$ \\
\hline \multicolumn{7}{|c|}{ Joint sensitivity correlation } \\
\hline
\end{tabular}

Estimates calculated at a significant probability $p^{*}<0.001$

Table 3. Nonlinear regression statistics

\begin{tabular}{cccc}
\cline { 2 - 4 } & Measures & Native model & Full model \\
\cline { 2 - 4 } & RMSE & 1.049 & 0.2233 \\
\hline$R_{p}^{2}$ & 0.9791 & 0.9978 \\
$\mathrm{AIC}$ & 3497.7 & $612.6^{\dagger}$ \\
$\mathrm{BIC}$ & 3512.9 & $647.9^{\dagger}$ \\
$\varepsilon(\%)$ & 30.16 & 11.43 \\
$\varepsilon_{m}(\%)$ & 13.73 & 6.25 \\
\hline \multicolumn{2}{c}{$N$} & 1500 & 150 \\
Statistical measures for native and full models. AIC & Parameters & 2 & 6 \\
\cline { 2 - 4 } & \multicolumn{3}{c}{ and BIC ${ }^{\dagger}$ values calculated using $N=1500$}
\end{tabular}

Nonlinear regression errors of the ten $K$-fold validations are presented in Table 4 . Each validation yielded a set of sub-parameters $n_{1}, n_{2}$, and $m_{1}$ calculated with a sample size $(N)$ of 1350 data points. The tabulated errors measures were obtained using these sub-parameters with the ten validation data-splits of the 150-point datasets. Notice the consistency between error measures in this Table (final model) and those in Table 3 (native model).

\subsection{Moving Boundary Model Performance}

Figure 5 compares FF time profiles predicted by Eq. (20), CNFD model, Eq. (2), Özisik's (1980) analytic solution, and Eq. (1), with the Biot number and sample levels of St and $\kappa$ as parameters. Though the full model's $\tau$ predictions are smaller than Plank's values, notably for low $\mathrm{Bi} \leq 0.1$, they are consistent with the CNFD model's $\tau$ values. Notice that for $\mathrm{Bi} \geq 40$, the full model's predictions agree with the Özisik solution, which assumes constant thermal properties and a Dirichlet BC.

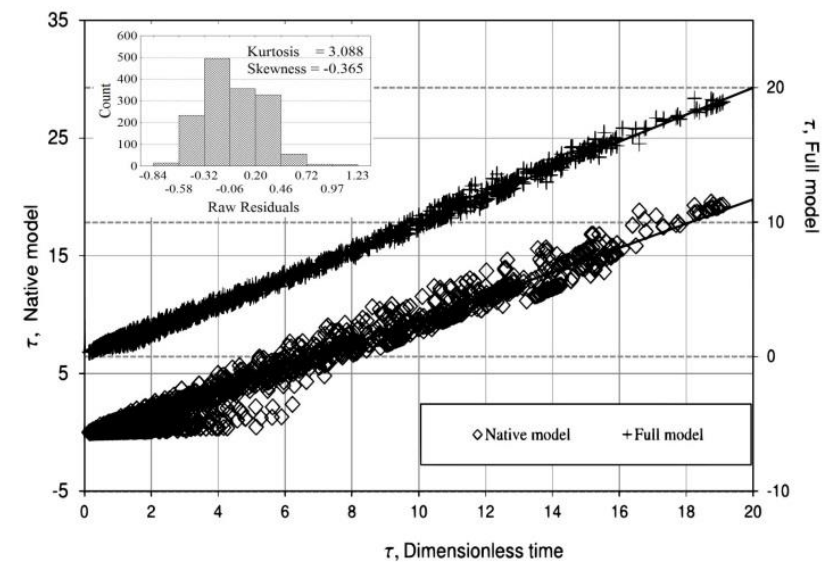

Figure 3. Comparison of native and full model fit responses vs. FF penetration times by the CNFD model (lower plot), and histogram of the raw residuals (upper-left corner plot) 


\subsection{Thermal Conductivity and Latent Heat Effects}

Relative errors in $\kappa(\delta \kappa / \kappa= \pm 6 \%)$ generate $(\mp \delta \hat{k} \hat{k})$ and $( \pm \delta \tau / \tau)$ uncertainties. These effects are illustrated as families of $s-\tau$ curves in Figure 6 with Bi as parameter. Although $\pm \delta \tau / \tau$ varies along the FF profile, its variability is insignificant $(F=0.145, p *=0.05)$. Figure 7 maps the sensitivity response against $\psi$ and $\mathrm{Bi} \cdot \kappa$. In the region limited by $(0.06<\mathrm{Bi} \cdot \kappa<2), \sigma_{\kappa}$ forms a narrow and unexpected pleat or fold (lower-right section of the map). The sensitivity values in this pleat are, nevertheless, positive and of the same magnitude of the stabilized sensitivity values for $\mathrm{Bi} \cdot \kappa>3$.

Descriptive statistics for $\sigma_{\kappa}$ and $\sigma_{\lambda}$ sensitivities are presented in Table 5. The three main-effects groups detected by Mathematica's GLM analysis in decreasing order of magnitude are:

$\{\kappa(F=1882.8)\}>\{\mathrm{Bi} \cdot \kappa(F=326.6), \mathrm{Bi} \cdot \psi(F=238.1), \psi(F=140.8), \psi \cdot \kappa(F=129.8)\}>\left\{\mathrm{Bi}^{2}(F=9.5)\right\}$,

where all $F$ values are significant at $p *<0.002$. Factors Bi and $\lambda$ independently exert no effect on $\sigma_{\mathrm{\kappa}}$.

Uncertainties in $\lambda(\delta \lambda / \lambda= \pm 3 \%)$ generate roughly similar errors $( \pm \delta \tau / \tau)$ in the FF penetration time shown (Figure 8 ), where families of $s-\tau$ profiles are presented with Bi as parameter but $\kappa$ and $\psi$ set at fixed levels. The mapped sensitivity $\left(\sigma_{\lambda}\right)$ response to $\lambda$ perturbations forms a concave surface with $\psi$ and Bi in Figure 9. Noteworthy is the $\sigma_{\lambda}$ decline for $0.06<\mathrm{Bi}<12$ and subsequent stabilization to levels that depend on $\psi$. GLM analysis identified the following three main-effects groups on $\sigma_{\lambda}$, which in decreasing order of magnitude, are:

$\{\mathrm{Bi}(F=6590)\}>\left\{\psi(F=2369), \mathrm{Bi} \cdot \psi(F=1784), \mathrm{Bi}^{2}(F=1356)\right\}>\{\kappa(F=70.9), \mathrm{Bi} \cdot \kappa(F=36.4)\}$,

where all $F$ values are significant at $p *<0.001$. Note that $\lambda$ uncertainties do not affect $\sigma_{\lambda}$.

A joint sensitivity $\left(\sigma_{\kappa}\right)$ range was estimated for test grid T1.3 introducing perturbations centered at $\kappa=0.5$ and $\lambda$ $=105$ for illustration purposes (Figure 10). Note the quick $\sigma_{\kappa \lambda}$ decay with increasing Bi and leveling off to $\sigma_{\kappa} \lambda$ values (almost linearly) depending on $\psi$. A statistical correlation to predict $\sigma_{\kappa \lambda}$ was developed as:

$$
\sigma_{\kappa \lambda}=\frac{c_{1}(1+\kappa)}{(1+\mathrm{Bi} \cdot \mathrm{St})^{0.5} c_{1}}+c_{2} \operatorname{Ln}[1+\mathrm{Bi}]
$$

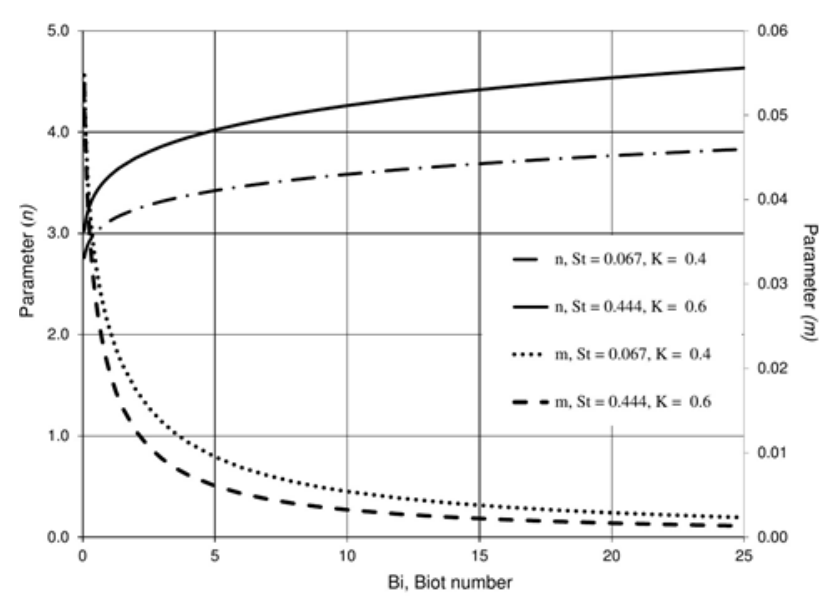

Figure 4. Effect of the Biot number on predicted values of temperature polynomial power $(n)$ and surface temperature parameter $(m)$, with $\mathrm{St}$ and $\kappa$ as parameters 
Table 4. $K$-fold validation results including mean $(\varepsilon)$, median $\left(\varepsilon_{m}\right)$ relative errors, and $R_{p}^{2}(N=150)$

\begin{tabular}{lclll}
\hline \multirow{2}{*}{$K$-fold training } & \multicolumn{4}{l}{ Statistical measures } \\
\cline { 2 - 5 } & $\mathrm{R}_{\mathrm{p}}^{2}$ & $\mathrm{RMSE}$ & $\varepsilon(\%)$ & $\varepsilon_{\mathrm{m}}(\%)$ \\
\hline $\mathrm{K} 1$ & 0.9995 & 0.329 & 12.71 & 5.36 \\
$\mathrm{~K} 2$ & 0.9962 & 0.285 & 11.41 & 5.53 \\
$\mathrm{~K} 3$ & 0.9960 & 0.282 & 10.66 & 4.93 \\
$\mathrm{~K} 4$ & 0.9951 & 0.287 & 10.02 & 4.58 \\
$\mathrm{~K} 5$ & 0.9962 & 0.295 & 12.35 & 5.89 \\
$\mathrm{~K} 6$ & 0.9967 & 0.286 & 11.72 & 5.23 \\
$\mathrm{~K} 7$ & 0.9946 & 0.349 & 12.97 & 5.36 \\
$\mathrm{~K} 8$ & 0.9963 & 0.282 & 10.90 & 5.44 \\
$\mathrm{~K} 9$ & 0.9964 & 0.268 & 13.09 & 5.25 \\
$\mathrm{~K} 10$ & 0.9961 & 0.311 & 12.52 & 5.48 \\
\hline Median & 0.9959 & 0.297 & 11.84 & 5.42 \\
\hline SD & 0.0007 & 0.025 & 1.06 & 0.35 \\
\hline Full model & 0.9983 & 0.297 & 11.83 & 5.36 \\
\hline
\end{tabular}

which is valid for $\hat{\varepsilon}_{\mathrm{K}}=\hat{\varepsilon}_{\lambda}$ and for grid T1.2 conditions. Nonlinear regression estimates and statistics describing this correlation are presented in Table 2 . The error measures supporting the fit include $R_{p}^{2}=0.9988$, RMSE $=$ 0.0248 , mean $\varepsilon=2.77 \%, \varepsilon_{m}=2.35 \%$, maximum intrinsic and parameter-effects curvatures are 0.00011 and 0.00044 , respectively, compared to a $95 \%$ curvature confidence-region of 0.57704 . The pairwise sensitivity coefficient $\left(c_{1}, c_{2}\right)$ is -0.3585 .

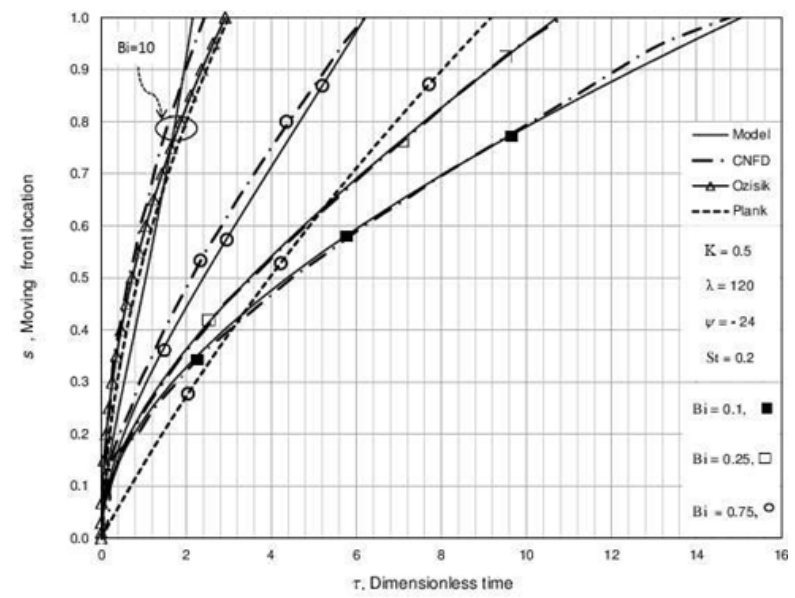

Figure 5. Comparison of freezing front predictions by Eq. (20) with selected published and CNFD freezing front models

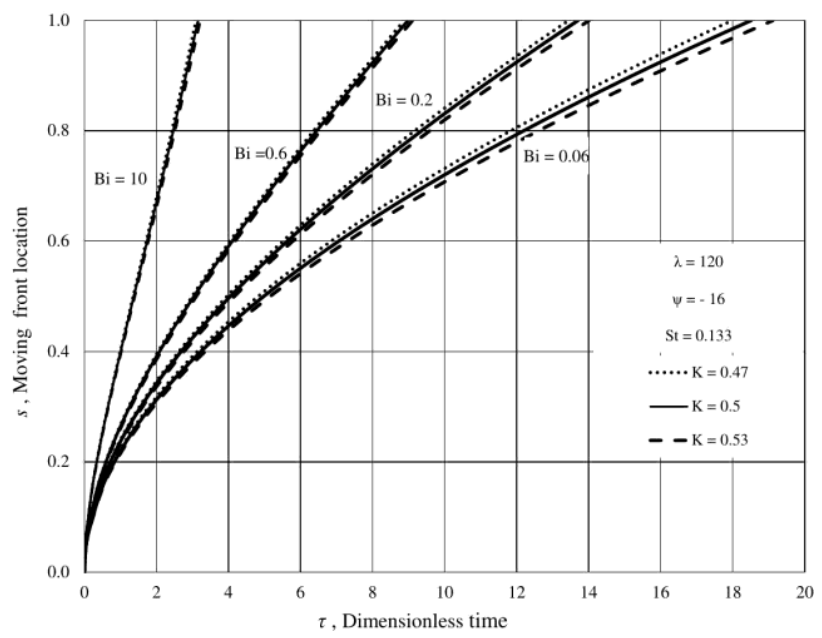

Figure 6. Effect of the conductivity parameter $\kappa( \pm 6 \%)$ deviations on the freezing front movement 
Table 5. Descriptive statistics of independent and joint sensitivities. MAD is $1.483 \times$ (median of absolute deviations)

\begin{tabular}{cccccc}
\hline \multicolumn{5}{c}{ Descriptive statistics } \\
\hline Sensitivity & Median & MAD & IQR & Minimum & Maximum \\
\hline$\sigma_{\mathrm{K}}$ & 0.348 & 0.152 & $0.220-0.425$ & 0.027 & 0.950 \\
$\sigma_{\lambda}$ & 0.967 & 0.032 & $0.877-0.985$ & 0.617 & 0.991 \\
$\sigma_{\mathrm{K} \lambda}$ & 0.664 & 0.086 & $0.611-0.712$ & 0.539 & 0.804 \\
\hline
\end{tabular}

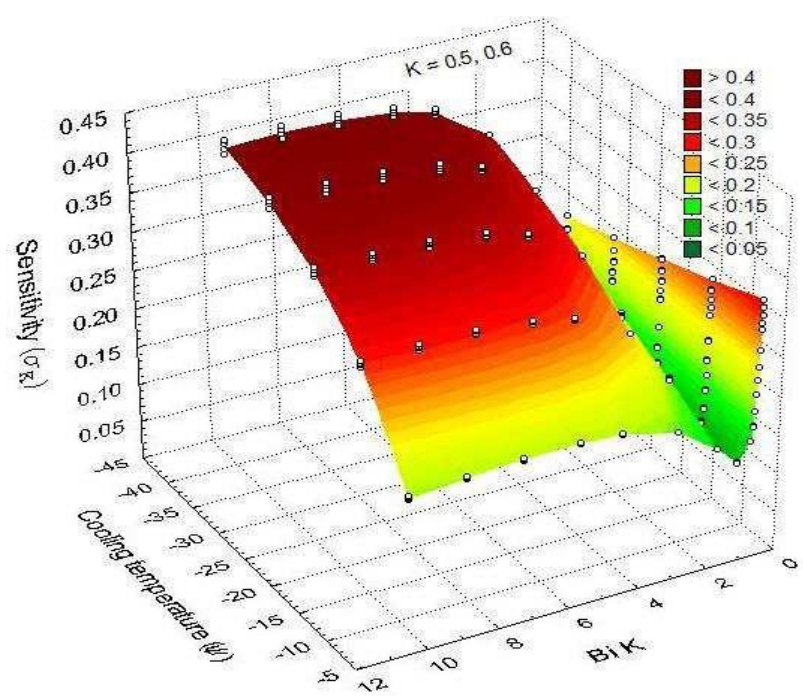

Figure 7. Sensitivity $\left(\sigma_{\mathrm{K}}\right)$ as influenced by Bi $\kappa$ and $\psi$, for $\kappa=0.5$ and 0.6 . Surface data smoothed by Statistica wafer-fit function

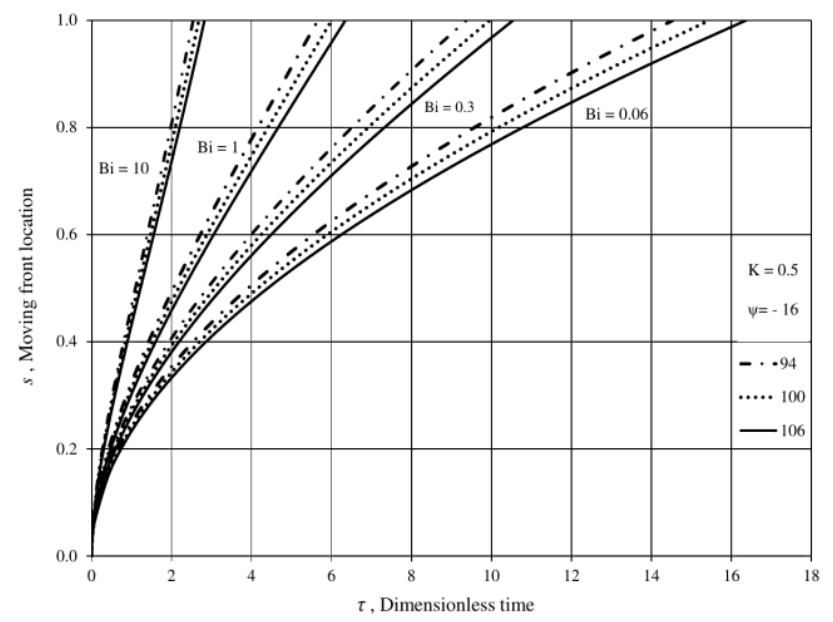

Figure 8. Effect of dimensionless latent heat $(\lambda)( \pm 3 \%)$ deviations on the freezing front movement

\section{Discussion}

Although numerical analysis enables solving food freezing problems with realistic assumptions, it may not be an efficient approach to quantifying the uncertainties introduced by estimated variable thermal properties (Özisik, 1980). We have thus developed an approximate analytical model to map these effects on the FF against relevant input properties, material and cooling conditions. The mentioned effects were calculated as sensitivities driven by independent or joint $\kappa$ and $\lambda$ uncertainties $\left(\sigma_{\kappa}, \sigma_{\lambda}\right.$, and $\sigma_{\kappa} \lambda$, respectively). Together, these sensitivities vary between 0.1 and 1 . 
For low to medium freezing rates and as $\mathrm{Bi}$ increases from 0.06 to approximately 6 , the $\kappa$-driven sensitivity range is $0.1 \leq \sigma_{\kappa} \leq 0.6$. Sensitivities $\sigma_{\lambda}$ and $\sigma_{\kappa \lambda}$, in turn, decrease from approximately 1 to $\leq 0.7$ and from roughly 0.8 to $\leq 0.55$, respectively. For high freezing rates $(\mathrm{Bi}>8)$, all sensitivities stabilize to levels controlled by $\psi$ for $\sigma_{\kappa}$ and $\sigma_{\kappa} \lambda$, and by $\psi \cdot \kappa$ for $\sigma_{\lambda}$. This means that the greater the cooling temperature $(\psi)$, the closer the thermal properties are to their frozen state values and the faster the sensitivities stabilize.

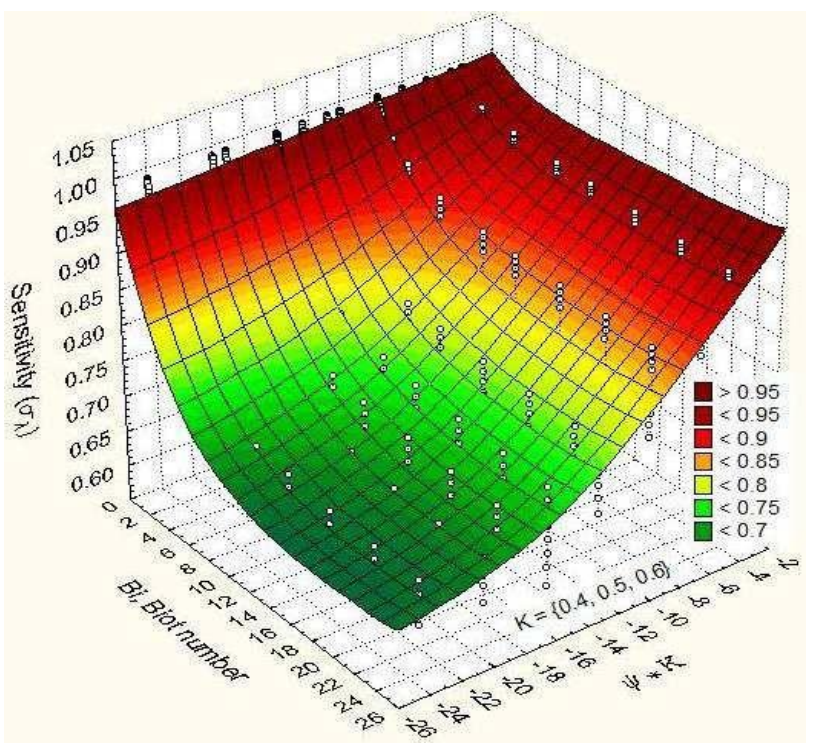

Figure 9. Dimensionless latent-heat driven sensitivity $\left(\sigma_{\lambda}\right)$ against Bi and $\psi \cdot \kappa$. Surface data smoothed by Statistica distance-weighted least-squares function

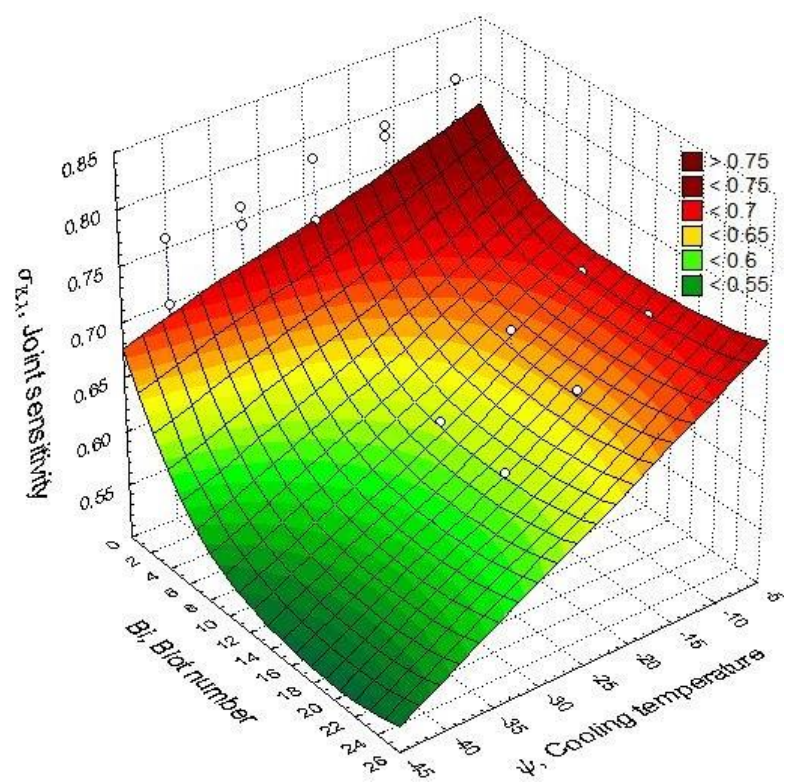

Figure 10. Effect of Bi and $\psi$ on the joint sensitivity $\left(\sigma_{\mathrm{\kappa} \lambda}\right)$. Surface data smoothed by Statistica negative exponential function

Lacking comparable sensitivity maps, we evaluate our $\sigma_{\mathrm{\kappa}}$ against Mittal et al.'s (1993) sensitivity values obtained from semi-empirical versions of Plank's Eq. (1). The median $\sigma_{\mathrm{K}}$ of $0.348(\mathrm{IQR}=0.205)$ is consistent with Mittal et al.'s absolute value range of $\mid-0.69$ to $-0.06 \mid$. Consider also that Plank's theoretical sensitivity relative to $k_{f}$ is $\bar{\sigma}_{k f}=-\mathrm{Bi} /(2+\mathrm{Bi})$. For high $\mathrm{Bi}>8, \sigma_{\mathrm{\kappa}}$ stabilizes to levels depending on $\psi \cdot \kappa$ (Figure 7$)$, which agrees with the theoretical $\bar{\sigma}_{k f}$ behavior against $\mathrm{Bi}$. There is no contradiction with the negative sign of Mittal et al.'s or Plank's sensitivities because relative deviations in the $\kappa$ drive changes of opposite sign in the fully frozen thermal 
conductivity $\left(k_{f}\right)$. Plank's mean $\left|\bar{\sigma}_{\mathrm{kf}}\right|$ is 0.794 (spanning from 0.029 to 0.926 ) in the Bi range of 0.06 to 25 . Note that $\sigma_{\kappa}<\bar{\sigma}_{k f}$. This is due to a Plank sensitivity that depends only on Bi, without accounting for either $\psi$ or any buffering effect of the Stefan number.

Our median $\sigma_{\lambda}(0.967, \mathrm{IQR}=0.107)$ is consistent with published values $(0.684$ to 1.172$)$ (Mittal et al., 1993$)$ and with the theoretical $\lambda$-driven Plank sensitivity of 1 . As the convection factors (Bi and $|\psi| \cdot \kappa)$ increase, $\sigma_{\lambda}$ drops at an ever-decreasing rate but stabilizes for Bi $>8$ at levels that depend on $\psi \cdot \kappa$. Therefore, the $\sigma_{\lambda}$ map reveals that the closer is the thermal layer to the fully frozen state or total heat removal, the smaller is the $\lambda$ uncertainty effect on the FF. Otherwise stated, the slower the convective freezing rate, the closer the FF is to mirror the $\lambda$ uncertainty.

The joint $\kappa$ and $\lambda$ median sensitivity $\left(\sigma_{\kappa}\right)$ falls between the independent $\sigma_{\kappa}$ and $\sigma_{\lambda}$ values. This result is consistent with the law of error propagation (Tellingheusen, 2001). Notice the dominating $\lambda$-perturbation influence on the $\sigma_{\kappa} \lambda$ response (Figures 9 and 10). Although the $\sigma_{\kappa} \lambda$ sensitivity correlation was developed for equal relative errors in $\kappa$ and $\lambda$, it yields a unique insight into the combined variable thermal property influence on the FF nevertheless. The odd $\sigma_{\kappa}$ pleat at the low end of $\psi$ and $\mathrm{Bi} \cdot \kappa$ ranges (i.e. mild cooling rate) stems from the balancing of the two rational terms containing the FF location $(s)$ in Eq. (20). Together they generate a slight inflection point in the first half of the FF. This subtle slope change becomes amplified at the end of the $s-\tau$ profile $(s=1)$, where $\sigma_{\kappa}$ is calculated. Beyond this inflection point, the $s-\tau$ curvature remains monotonic.

Several HBI analyses of simplified Stefan problems have either assumed or estimated the temperature polynomial power ( $n$ ) from known analytical solutions (Goodman, 1958; Özisik, 1980; Mitchell \& Myers, 2012). For a realistic problem formulation, neither case applies. However, it is more coherent with the phase change physics to extract $n$ and $m$ information from the FF data via nonlinear regression. This unique approach enabled us to estimate constant $\hat{n}$ and $\widehat{m}$ but, more importantly, of $n$ and $m$ correlations with the input data ( $\mathrm{Bi}, \psi, \lambda$, and $\kappa)$. The lack of comprehensive, structured, and reliable experimental FF data justified generating these by a numerical solution of the energy equation.

Whereas our constant $\hat{n}$ estimate is 3.08 , the adjustable $n$ correlation predicted values ranging from approximately 2.8 to 5.3 depending on $\mathrm{Bi}$, St, and $\psi$ levels. In comparison, Mitchell and Myers' (2012) HBI analysis yields $\hat{n} \approx 3.6$ assuming constant properties. As Bi increases above 8 , the shift of $n$ towards a quintic power is consistent with Schwartzberg's (1977) freezing-simulation temperature profiles. The higher the $n$ value above 2, the more pronounced is the temperature profile convexity with a plateaued top. A drawback of the temperature polynomial assumption is that, for Bi values below 0.1 , the temperature profile cannot plateau unless $n$ is less than 1, which violates the energy balance at the interface. Last, if $n$ is defined as time dependent (Mitchell \& Myers, 2012), the solution would most likely require numerical methods, preventing thus an analytical expression for the FF.

The parameter $m$ was introduced into Eq. (13) to modulate the convective Bi effect. An $m$ parameter magnitude of $\mathcal{O}\left[S t=10^{-1}\right]$ was expected because, in phase change analysis, St reduces the Bi convective effect. Indeed, for most foodstuffs, St values range between 0.068 and 0.444 (Schwartzberg, 1977). To develop Eq. (22), we conjectured that $m$ would correlate with $\mathrm{Bi}$, St, and $\kappa$. First, a power term of Bi could enhance the first-order rational $\mathrm{Bi}$ expression in Eq. (13). Second, Eq. (13) misses the buffering effect $\mathrm{St}$ has on $\mathrm{Bi}$, and thus the incorporation of the Stefan number. Third, for Bi $>0.1$ the thermal conductivity (via $\kappa$ ) should influence the slab temperature profile.

All nonlinear regression statistics in Tables 2 to 4 show significant reductions in parameter, curvature, and regression error measures. The (small magnitude of the intrinsic and parameter-effects) curvature measures relative to the $95 \%$ curvature confidence-region confirm the asymptotic and almost linear behavior of the sub-parameters at their converged values. Parameter reliability is thus corroborated (Karolczak \& Mickiewicz, 1995; Seber \& Wild, 2003).

Whereas the full model is in close agreement with the numerical simulation FF penetration time values because of the fit, it is also consistent with Özisik's (1980) analytic solution for Bi values above 8. Plank's predictions are consistently higher than our predictions because they are based on the complete heat release at the initial freezing point. The robust statistical measures supporting the improved fit quality include the minimized AIC, BIC, RMSE, mean and median relative errors $\left(\varepsilon\right.$ and $\left.\varepsilon_{m}\right)$, and $R_{p}^{2}$ (Table 3). The median relative regression error $\left(\varepsilon_{m}\right)$ was reduced by half to $5.36 \%$. These error measures and graphical assessment of the fit response vs. FF data together endorse the final model accuracy.

Though the normality tests give mixed results, the distribution graph of residuals is not radically non-normal. The large sample size of the FF data supports the reliability of the estimates under the central limit theorem 
(Beck \& Arnold, 1977; Ghasemi \& Zahediasl, 2012). Our FF model accuracy is based on conservative error measures (Tables 3 and 4) that are averages of ten $K$-fold data splits and not of the whole dataset (Jung \& Hu, 2015).

Together, the robustness of our FF model and sensitivity analysis are supported by (a) the physical significance of property parameters $(\kappa$ and $\lambda$ ), (b) final model accuracy, (c) consistent predictions with published average freezing-time sensitivities, and (d) coherent patterns for high Biot numbers and cooling temperature. Our sensitivity maps may be applied to the cooling and subcooling freezing process stages. Particular attention must be given to error estimation for low to medium freezing rate processes $(\mathrm{Bi}<8)$, because of the rapid sensitivity change with the Biot number and cooling temperature $(\psi$ or $\psi \cdot \kappa)$.

Future studies could invoke alternative convective-surface temperature models (relaxing the PSS assumption) that account for temperature-dependent thermal properties. These models should lead to consistently monotonic $s-\tau$ profiles without anomalies. Time dependence of the temperature polynomial power $(n)$ could be pursued, but it may lead to an irreducible energy PDE. This investigation could also be extended to other regular geometries and account for a temperature-dependent density.

\section{Conclusions}

Our approximate model enabled us to quantify and map systematically the effects of temperature-dependent thermal properties on the FF with acceptable accuracy. We show that the Biot number, cooling temperature, and thermal conductivity markedly and nonlinearly control these FF effects. While the thermal conductivity effect exhibits the greatest variability, the dimensionless latent heat effect is greater and essentially generates a direct and proportional perturbation on the FF. Nonlinear methods and prior information can thus be effective in constructing reliable mathematical models for phase change food processes together with its parameterization via nonlinear regression.

\section{List of Symbols}

$\begin{array}{ll}\text { AIC } & \text { Akaike information criterion } \\ \text { Bi } & \text { Biot number, } \mathrm{h} L / k_{f} \\ \text { BIC } & \text { Bayesian information criterion } \\ c_{p} & \text { volumetric heat capacity below } T_{i}, \text { dimensional } \\ \widehat{c_{p}} & \text { dimensionless effective and volumetric heat capacity below } T_{i},\left(c_{p} / c_{p f}\right) \\ \mathrm{h} & \text { surface heat transfer coefficient, dimensional } \\ \Delta \mathrm{H} & \text { latent heat of moist food material, at the initial freezing point, adjusted by bound water content, } \\ & \text { dimensional } \\ \hat{k} & \text { thermal conductivity below } T_{i}, \text { dimensional } \\ N & \text { dimensionless thermal conductivity, }\left(k / k_{f}\right) \\ L & \text { sample size } \\ R^{2} & \text { slab half thickness, dimensional } \\ R_{p}^{2} & \text { coefficient of determination } \\ \text { RMSE } & \text { coefficient of determination adjusted for number of parameters } \\ \text { St } & \text { root means square error } \\ t & \text { Stefan number, }-\psi /\left(c_{p}\left(T_{i}-T_{a}\right)\right) \\ T & \text { dimensional time } \\ u & \text { dimensional food slab temperature } \\ v & \text { Kirchhoff-transformed temperature, dimensionless } \\ X & \text { re-scaled dimensionless temperature, }\left(T-T_{a}\right) /\left(T_{o}-T_{i}\right) \\ x & \text { dimensional freezing front or moving boundary location } \\ & \text { dimensional spatial coordinate }\end{array}$




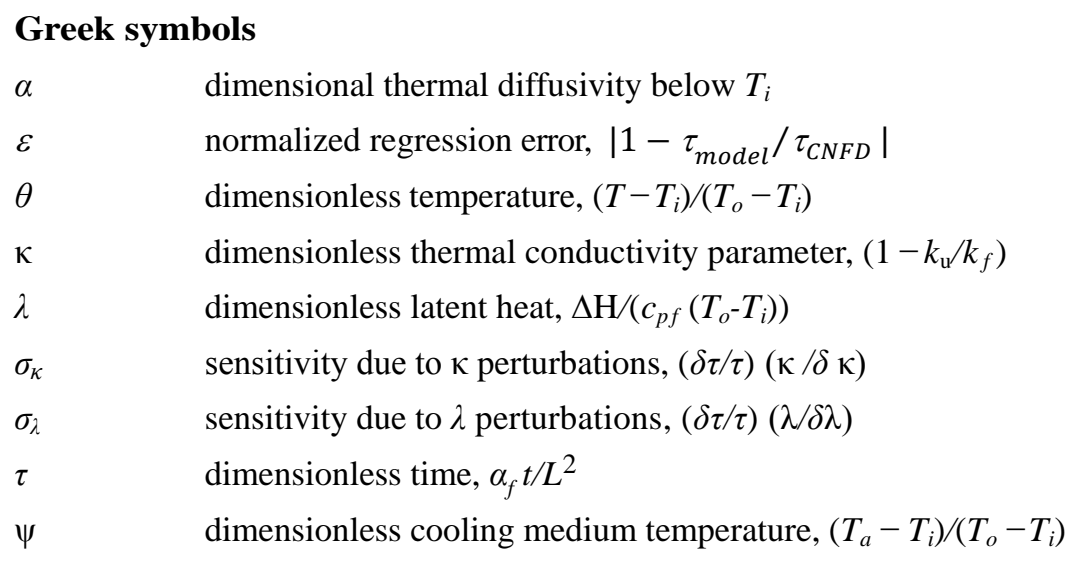

\section{Subscripts}

a external cooling medium

$f \quad$ fully frozen state

$i \quad$ initial freezing point

m median

o freezing point of pure water

$u \quad$ unfrozen food state

w cooled surface

\section{Acknowledgments}

VMCh is grateful for the insights regarding nonlinear analysis of Dr. Alexey Brobick, Department of Astrophysics at the University of Lund, Sweden.

Funding: This research received no external funding.

Conflict of interest: The author declares no conflict of interest.

\section{References}

Arboretti-Giancristofaro, R., \& Salmaso, L. (2003). Model performance analysis and model validation in logistic regression. Statistica, 2, 375-396. https://doi.org/10.6092/issn.1973-2201/358

Archontoulis, S., \& Miguez, F. (2015). Nonlinear regression models and applications in agricultural research. Agronomy Journal, 102(2), 786-796. https://doi.org/10.2134/agronj2012.0506

Baudouy, B. (2002). Integral method for transient HE II heat transfer in a semi-infinite medium. AIP Conference Proceedings, 613, 1349-1355. https://doi.org/10.1063/1.1472164

Bazant, M. (2018). Ch. 2: Pseudosteady approximation: moving boundary. In Analysis of transport phenomena I: Mathematical methods. Retrieved from https://courses.edx.org/courses/course-v1:MITx+10.50.1x+3T2018/course/

Beck, J., \& Arnold, K. (1977). Parameter estimation in engineering and science. New York, NY: John Wiley \& Sons.

Bellera, C., \& Hanley, J. (2007). A method is presented to plan the required sample size when estimating regression-based reference limits. Journal of Clinical Epidemiology, 60, 610-615. https://doi.org/10.1016/j.jclinepi.2006.09.004

Carnahan, B., Luther, H., \& Wilkes, J. (1969). Applied numerical methods. New York, NY: John Wiley \& Sons.

Chavarria, V. M. (2019). Food-slab freezing front data [Data set]. Harvard Dataverse, V1. https://doi.org/10.7910/DVN/LKTEJQ

Cho, S., \& Sunderland, J. (1974). Phase change with temperature-dependent thermal conductivity. Journal of Heat Transfer - Transactions of the ASME, 96, 214-217. https://doi.org/10.1115/1.3450167

Cottrell (2006). Temperature measurement. Retrieved from https://web.mst.edu/ cottrell/ME240/Resources/Temperature/Temperature.pdf 
Delgado, A., \& Sun, D.-W. (2001). Heat and mass transfer models for predicting freezing processes: A review. Journal of Food Engineering, 47(3),157-174. https://doi.org/10.1016/S0260-8774(00)00112-6

Farid, M. (2002). The moving boundary problems from melting and freezing to drying and frying of food. Chemical Engineering and Processing, 41, 1-10. https://doi.org/10.1016/S0255-2701(00)00153-7

Fricke, B., \& Becker, B. (2001). Evaluation of thermo-physical property models for foods. Heating, Ventilation and Air Conditioning, 7(4), 311-330. https://doi.org/10.1080/10789669.2001.10391278

Ghasemi, A., \& Zahediasl, S. (2012). Normality tests for statistical analysis: A guide for non-statisticians. International Journal of Endocrinology and Metabolism, 10, 486-499. http://dx.doi.org/10.5812/ijem.3505

Goodman, T. (1958). The heat-balance integral method and its application to problems involving a change of phase. Transactions of the American Society of Mechanical Engineers, 80, 335-342.

Heldman, D., \& Singh, R. (1981). Food Process Engineering (2 ${ }^{\text {nd }}$ ed.) Westport, CT: AVI. https://doi.org/10.1007/978-94-010-9337-8

Hu, H., \& Argyropoulos, S. (1996). Mathematical modeling of solidification and melting: A review. Modeling and Simulation in Material Science and Engineering, 4, 371-396. https://doi.org/10.1088/0965-0393/4/4/004

Jung, Y., \& Hu, J. (2015). A $K$-fold averaging cross-validation procedure. Journal of Nonparametric Statistics, 27(2), 167-179. https://doi.org/10.1080/10485252.2015.1010532

Karolczak, M., \& Mickiewicz, A. (1995). Why to calculate, when to use, and how to understand curvature measures of nonlinearity. Current Separations, 14, 10-16.

Kucera, A. (1985). Mathematics of moving boundary problems in diffusion (Doctoral dissertation, University of Wollongong, Australia). Retrieved from http://ro.uow.edu.au/theses/1553

Kumar, A., \& Singh, R. (2018). A moving boundary problem with variable specific heat and thermal conductivity. Journal of King Saud University of Science, 1-6. http://dx.doi.org/10.1016/j.jksus.2018.05.28

López-Leiva, M., \& Hallström, B. (2003). The original Plank equation and its use in the development of food freezing rate predictions. Journal of Food Engineering, 58(3), 267-275. https://doi.org/10.1016/S0260-8774(02)00385-0

Lunardini, V. (1988). Heat conduction with freezing or thawing (Monograph 88-1). Hanover, NH: U.S. Army Cold Regions Research and Engineering Laboratory.

Mitchell, S., \& Myers, T. (2012). Application of heat balance integral methods to one-dimensional phase change problems. International Journal of Differential Equations. https://dx.doi.org/10.1155/2012/187902

Mittal, G., Hanenian, R., \& Mellikarjunan, P. (1993). Evaluation of freezing time prediction models for meat patties. Canadian Agricultural Engineering, 35(1), 75-81.

Özisik, M. (1980). Heat Conduction. New York, NY: John Wiley \& Sons.

Pham, Q. (1986). Simplified equation for predicting the freezing time of foodstuffs. International Journal of Food Science \& Technology, 21(2), 209-219. https://doi.org/10.1111/j.1365-2621.1986.tb00442.x

Pham, Q., \& Willix, J. (1990). Effect of Biot number and freezing rate on accuracy of some food freezing time prediction methods. Journal of Food Science, 55(5), 1429-1434. https://doi.org/10.1111/j.1365-2621.1990.tb03951.x

Rajeev, R. (2014). Homotopy perturbation method for a Stefan problem with variable latent heat. Thermal Science, 18(2), 391-398. https://doi.org/10.2298/TSCI110627008R

Ramos, M., Cerrato, Y., \& Gutierrez, J. (1994). An exact solution for the finite Stefan problem with temperature-dependent thermal conductivity and specific heat. International Journal of Refrigeration, 17(2), 130-134. https://doi.org/10.1016/0140-7007(94)90054-X

Rao, M., Rizvi, S., \& Datta, A. (2005). Engineering properties of foods (3rd ed.). Boca Ratón, FL: CRC Press.

Schwartzberg, H. (1976). Effective heat capacities for the freezing and thawing of food. Journal of Food Science, 4l(1), 152-156. https://doi.org/10.1111/j.1365-2621.1976.tb01123.x

Schwartzberg, H. (1977). The prediction of freezing and thawing temperature vs. time through the use of effective heat capacity equations. Proceedings of the Joint Meeting of Commissions C1 and C2. International Institute of Refrigeration, 311-317. 
Schwartzberg, H., Singh, R., \& Sarkar, A. (2007). Freezing and thawing - Computation methods and thermal properties correlation. In S. Yanniotis \& B. Sunden (Eds.), Heat transfer in Food Processing. https://doi.org/10.2495/978-1-85312-932-2/03

Seber G., \& Wild, C. (2003). Nonlinear Regression. Hoboken, NJ: John Wiley \& Sons.

Singh, J., Gupta, K., \& Ral, K. (2011). Variational iteration method to solve moving boundary problem with temperature dependent physical properties. Thermal Science, 15(2), 229-239. https://doi.org/10.2298/TSCI100226024S

Solomon, A., Wilson, D., \& Alexiades, V. (1981). The quasi-stationary approximation for the Stefan problem with convective boundary condition (Technical Report ORNL/CSD-84). Oak Ridge, TN: Oak Ridge National Laboratory. https://doi.org/10.2172/6218971

Tellingheusen, J. (2001). Statistical error propagation. Journal of Physical Chemistry-US, 105, 3917-3921. https://doi.org/10.1021/jp003484u

Voller, V., Swenson, J., \& Paola, C. (2004). An analytical solution for a Stefan problem with variable latent heat. International Journal of Heat and Mass Transfer, 47(24), 5387-5390. https://doi.org/10.1016/j.ijheatmasstransfer.2004.07.007

Wood, A. (2001). A new look at the heat balance integral method. Applied Mathematical Modeling, 25, 815-824. https://doi.org/10.1016/S0307-904X(01)00016-6

\section{Copyrights}

Copyright for this article is retained by the author(s), with first publication rights granted to the journal.

This is an open-access article distributed under the terms and conditions of the Creative Commons Attribution license (http://creativecommons.org/licenses/by/4.0/). 